\title{
Unexpected Emission Properties of a 1,8-Naphthalimide Unit Covalently Appended to a Zn-Salophen
}

Martina Piccinno,[a][b] Gemma Aragay,[b] Francesco Yafteh Mihan,[a] Pablo Ballester, ${ }^{*}[b][c]$ and Antonella Dalla Cort*[a]

Abstract: We report the synthesis, characterization and binding properties of a Zn-salophen complex, 1a, functionalized with a 1,8-naphthalimide unit. Unexpectedly, the emission spectrum of 1a shows a remarkable quenching of the band assigned to the naphthalimide unit. To better understand this phenomenon, a supramolecular model system constituted by a symmetric $\mathrm{Zn}$-salophen and a pyridyl derivative of 1,8-naphthalimide, $1 \mathrm{~b} \bullet 2 \mathrm{a}$, is investigated. We propose the existence of a photoinduced energy transfer process between the naphthalimide (donor) and the salophen (acceptor) units in $1 \mathrm{~b} \cdot 2 \mathrm{a}$. A similar process must be operative in the covalent receptor $1 \mathrm{a}$. Nevertheless, the results deriving from steady-state fluorescence experiments do not rule out the occurrence of a photoinduced electron transfer process as alternative pathway for the quenching. We also describe the chemosensing properties of the receptor $1 \mathrm{a}$ and the supramolecular system $1 \mathrm{~b} \bullet 2 \mathrm{a}$ towards acetate. The nonsymmetrically substituted salophen receptor 1 a only transduces the binding of the anion to the $\mathrm{Zn}$-metal centre in significant spectroscopic changes in its absorption spectrum. On the other hand, we exploit the strong emission quenching experienced by the naphthalimide component in the supramolecular complex $1 \mathrm{~b} \bullet 2 \mathrm{a}$ to detect anions (e.g. acetate) by means of a typical "turn-on" fluorescent indicator-displacement assay.

\section{Introduction}

The importance and ubiquity of anions in many chemical and biological processes have boosted the field of anion coordination chemistry research. ${ }^{1,2,3}$ Anions are not only important in many sustaining life processes (i.e. enzymatic processes) but also represent a serious environmental problem (e.g. eutrophication).

Many elegant studies have been reported dealing with the development of optical sensors for anions and the understanding of their binding modes and sensing mechanisms. ${ }^{1,4,5}$ of particular interest are optical molecular probes based on a recognition site covalently linked to a chromophore/fluorophore unit. ${ }^{6,7,8}$ In these systems, the recognition unit binds selectively the target species and the molecular recognition event is transduced through the modulation of the optical properties of the signaling unit (chromophore). For decades, chromogenic/absorbing organic dyes have been widely used as signaling units. Nowadays, however, fluorescent dyes are preferred because they provide increased sensitivity to the sensing methodology. . $^{5}$

In the last years, zinc-salophen complexes have attracted increasing attention owing to the Lewis acidity of the $\mathrm{Zn}$ (II) center that makes them excellent receptors for electron-rich substrates including neutral molecules and anions ${ }^{10}$. Such interesting properties come along 
with an easy synthetic access and reasonable chemical stability. The recognition event is generally monitored by absorption spectroscopy because the quantum yield of the $\mathrm{Zn}$ salophen fluorescence is usually very low. ${ }^{11,12}$ We envisaged that the decoration of the $\mathrm{Zn}$ salophen scaffold with a highly fluorescent unit must produce a chemosensor able to signal the binding of target molecules to the Zn-center through changes in the emission properties of the attached chromophore. Our goal was to increase the sensibility of the sensing system by changing the transduction of the binding event from absorption to fluorescence signaling. We focused our attention on the 1,8-naphthalimide fluorophore as reporter unit. The 1,8naphthalimide unit is characterized by unique photophysical properties (i.e. high quantum yield), and straightforward tunability by simple modifications of the naphthalene skeleton and/or at the imide unit. ${ }^{13}$ 1,8-naphthalimide based receptors covalently linked to Zn2+ complexes have already been reported in the literature. ${ }^{14,15,16}$ Recognition of an anion or a cation by the binding center is able to modulate the emission properties of the naphthalimide fluorophore, giving rise to a signaling optical response.

Herein, we report the synthesis and characterization of a non-symmetrically substituted Znsalophen having one 1,8-naphthalimide unit tethered by a triazole ring (1a, Figure 1).

Figure 1.

The presence of two tert-butyl groups in one half of the salophen unit of 1a was aimed to prevent its dimerization through intermolecular $\mathrm{Zn}-\mathrm{O}$ interactions ${ }^{17}$. On the other hand, the $\mathrm{N}$-octyl chain introduced in the naphthalimide component served to increase the solubility of $1 \mathrm{a}$ in organic solvents. We investigated the absorption and emission properties of $1 \mathrm{a}$, as well as its binding properties towards acetate anion that was used to test the behavior of the system in the presence of coordinating anions. Our studies revealed unexpected features in the emission properties of 1 a which stopped us to further evaluate the effect that the coordination of other anions produced on the system. To rationalize the obtained results, we designed and studied a supramolecular model system based on the pyridine naphthalimide derivative $2 \mathrm{a}$ and the symmetrically substituted $\mathrm{Zn}$-salophen $1 \mathrm{~b}$. In solution, these components assembled into a 1:1 complex through axial coordination of the pyridine group of 2a with the $\mathrm{Zn}$ metal center of $1 \mathrm{~b}$. We describe the spectroscopic and thermodynamic characterization of the $1 \mathrm{~b} \bullet 2 \mathrm{a}$ complex. The obtained results with the supramolecular model $1 \mathrm{~b} \bullet 2 \mathrm{a}$ indicated that the quenching of the naphthalimide emission was caused by a photoinduced process that involved the $\mathrm{Zn}$-salophen component. This result also explains the unexpected emission properties found for the naphthalimide unit in the covalently connected receptor $1 \mathrm{a}$.

\section{Results and Discussion}

Synthesis and characterization of Zn-salophen 1a

5-(azidomethyl)-2-hydroxybenzaldehyde 3 was obtained by reacting sodium azide with 5(chloromethyl)-2-hydroxybenzaldehyde (See SI). On the other hand, commercially available 4Bromo-1,8-naphthalic anhydride was first converted to the corresponding imide derivative. Then, an ethynyl sustituent was introduced by Sonogashira coupling followed by deprotection of the trimethylsilyl group to finally afford the ethynyl-naphthalimide derivative 4 (See SI). $\mathrm{Cu}(\mathrm{I})$ catalyzed reaction of the azide salycilaldehyde derivative 3 with alkyne 4 afforded the 
1,8-naphthalimide triazole 5 in $69 \%$ yield after column chromatography purification. The condensation reaction of monoimine 6 with the functionalized salycilaldehyde 5 produced $\mathrm{Zn}$ salophen 1a as an orange solid in $75 \%$ yield (Scheme 1 ). Salophen 1a was characterized by a full set of high resolution spectra (see SI).

Scheme 1.

The $1 \mathrm{H}$ NMR spectrum of salophen $1 \mathrm{a}$ in $\mathrm{CDCl} 3$ solution displayed broad signals for the aromatic protons of the salophen core. Most likely, the broadening of the signals is caused by a chemical exchange process between monomer and dimer forms of $1 \mathrm{a}$ that occurred at an intermediate rate on the chemical shift timescale. The tendency of $\mathrm{Zn}$-salophen derivates to dimerize at millimolar concentration in weakly or non-coordinating solvents is well-known. ${ }^{17}$

Conversely, the $1 \mathrm{H}$ NMR spectrum of $1 \mathrm{a}$ in DMSO-d6 solution showed sharp and well-defined proton signals, which were easily assigned using 2D NMR experiments (Figure S5 and S6 SI). The imine protons $\mathrm{H} 3$ and $\mathrm{H} 8$ resonated separately at $\delta=8.99$ and $8.94 \mathrm{ppm}$ respectively, confirming the non-symmetrical nature of the $\mathrm{Zn}$-salophen core. Moreover, the triazole proton $\mathrm{H} 13$ appeared as a sharp singlet at $\delta=8.89 \mathrm{ppm}$. NOE cross-peaks were observed between the triazole proton $\mathrm{H} 13$ and aromatic protons $\mathrm{H} 14$ and $\mathrm{H} 18$ present in the naphthalimide unit. This is an expected result if we consider the free rotation of the $\mathrm{C}-\mathrm{C}$ bond connecting the triazole ring and the 1,8-naphthalimide unit.

A DOSY experiment performed on a DMSO-d6 solution of 1a at $298 \mathrm{~K}$ (Figure S7 SI) assigned a diffusion coefficient of

$1.41 \times 10-10 \mathrm{~m} 2 / \mathrm{s}$ to the molecule. This diffusion coefficient value corresponds to a hydrodynamic radii (rexp) of $7.66 \AA$, which is in good agreement with the dimensions of the molecule estimated using molecular modeling (Figure 2). ${ }^{18}$

Figure 2.

\section{Solution binding studies}

Thermodynamic characterization of the complex of 1a with acetate anion.

Zinc-salophens are known to be good receptors for anions. ${ }^{19,20}$ Preliminary studies to examine the behavior of $1 \mathrm{a}$ in the presence of an anion were performed with acetate.

The interaction of $1 \mathrm{a}$ with acetate was probed using $1 \mathrm{H}$ NMR spectroscopy (Figure 3 ). The addition of incremental amounts of tetrabutylammonium acetate, TBA(AcO), to a ${ }^{1} \mathrm{mM}$ solution of $1 \mathrm{a}$ in $\mathrm{CDCl} 3$ resulted in chemical shift changes of its proton signals and the sharpening of some of them that initially were broad.

Figure 3. 
This observation confirmed that the acetate anion coordinated to the zinc metal center of the salophen core of $1 \mathrm{a}$ and disrupted its dimerization. The addition of more than 1 equivalent of acetate did not induce any additional change in the NMR spectra. We concluded that a 1:1 complex $1 \mathrm{a} \bullet \mathrm{AcO}$ - is formed with an association constant higher than $104 \mathrm{M}-1$. In order to assess an accurate stability constant value for the $1 \mathrm{a} \bullet \mathrm{AcO}$ - complex we next performed a UVVis titration experiment.

The UV-Vis spectrum of the zinc-salophen 1a in chloroform solution displayed two broad absorption bands with maxima centered at $300 \mathrm{~nm}$ and $365 \mathrm{~nm}$.

This spectrum closely resembles the sum of the absorption profiles of the two separated chromophores in $1 \mathrm{~b}$ and $2 \mathrm{~b}$. This result suggested us that the electronic interaction of the two chromophores in the ground state of $1 \mathrm{a}$ is weak (Figure 4).

Figure 4.

A dilution experiment of $1 \mathrm{a}$ in a range of concentrations of $10^{-7} \div 10^{-5} \mathrm{M}$ showed that the absorption spectra in epsilon scale remained constant (Figure S15 SI). Hence, in complete agreement with a previous report for a related system we concluded that in this range of concentrations 1a should exist in solution as a monomeric species.

The addition of increasing amounts of TBA(AcO) salt (0-6 eq) to a $10^{-5} \mathrm{M}$ chloroform solution of 1a caused the decrease of the absorption bands centered at 300 and $365 \mathrm{~nm}$ and the simultaneous appearance of a new band with a maximum at $405 \mathrm{~nm}$ (Figure 5).

Figure 5.

The titration spectra provided a clear isosbestic point centered at $380 \mathrm{~nm}$ suggesting the existence in solution of a single equilibrium involving the two species. Accordingly, the UV-Vis titration data were analyzed using a theoretical 1:1 binding model that considered two colored species (free $1 \mathrm{a}$ and $1 \mathrm{a} \bullet \mathrm{AcO}$ - complex). The fit was good and returned an association constant value of $\mathrm{Ka}=(1.73 \pm 0.10) \times 105 \mathrm{M}-1$ for the $1 \mathrm{a} \bullet \mathrm{AcO}$ - complex. The determined association constant value is in agreement with those reported for analogous zinc-salophen receptors binding acetate anion ${ }^{19}$

Emission spectroscopy studies of salophen $1 a$. 
The decoration of the salophen complex with the 1,8-naphthalimide unit was expected to improve the spectroscopic emission properties of the system and allow its use at low concentrations (10-6 - 10-7 M) to detect the presence of anions. To test this hypothesis we decided to study the complexation of acetate ion by $1 \mathrm{a}$ at micromolar concentration using emission spectroscopy.

Figure 6 shows the emission spectrum in chloroform of $1 \mathrm{a}(\lambda \mathrm{exc}=360 \mathrm{~nm})$. Two broad emission bands centered at 420 and $620 \mathrm{~nm}$, respectively, are visible. By comparison with the emission spectra of $1 \mathrm{~b}$ and $2 \mathrm{~b}$ we attributed them to separate emission of the two chromophores present in the structure of 1a: the 1,8-naphthalimide $(420 \mathrm{~nm})$ and $\mathrm{Zn}$-salophen $(620 \mathrm{~nm})$.

However, the 1,8-naphthalimide chromophore in $2 \mathrm{~b}$ is a significantly better emitter than zincsalophen chromophore of $1 \mathrm{~b}$, see Figure 6 . In fact, the emission quantum yield (Фem) of the 1,8-naphthalimide chromophore $2 \mathrm{~b}$ was measured to be 0.8 , much higher than the reported value for the zinc-salophen complex $1 b$ that was described to be $\Phi$ em $=0.3 .^{22}$ Notably, the high emission exhibited by the 1,8-naphthalimide in $2 \mathrm{~b}$ is strongly quenched when the chromophore is covalently incorporated in the structure of $1 \mathrm{a}$. This result indicates the existence of a quenching mechanism for the emission of the naphthalimide unit in 1a, i.e. photoinduced electron transfer (PET) or energy transfer (ET).

Figure 6.

The emission spectrum of 1 a showed quite similar intensities for the bands assigned to the 1,8naphthalimide and zinc-salophen units which did not match with the different quantum yield observed for the two separated units. The observed unexpected quenching in the emission of the 1,8-naphthalimide covalently attached to the $\mathrm{Zn}$-salophen in 1a prompted us to further investigate the system by using a model in which the two chromophores were kept close in space through a coordination bond, yielding a supramolecular complex.

\section{Synthesis of the components of the supramolecular complex. Thermodynamic characterization of the complex.}

The designed supramolecular system is based on the symmetrically substituted $\mathrm{Zn}$-salophen $1 \mathrm{~b}$ and pyridine derivative 2 a having a 1,8-naphthalimide unit (Figure 1 ). The $\mathrm{Zn}$-salophen $1 \mathrm{~b}$ was synthesized following a procedure reported in literature ${ }^{23}$. Naphthalimides $2 a$ and $2 b$ were prepared by $\mathrm{Cu}(\mathrm{I})$ catalyzed reactions of $\mathrm{N}$-ethyl-6-ethynil-1,8-naphthalimide S1 with 4(azidomethyl)pyridine S2 or benzyl azide S3, respectively (See SI).

Compounds $1 \mathrm{~b}$ and $2 \mathrm{a}$ are expected to self-assemble in solution through axial coordination of the nitrogen atom of the pyridine-ligand to the $\mathrm{Zn}$ metal center. Based on literature precedents the association constant for the assembly $1 \mathrm{~b} \cdot 2 \mathrm{a}$ was estimated as $K_{a} \sim 10^{5} \mathrm{M}^{-}$

${ }^{1.24}$ Derivative $2 \mathrm{~b}$ was used as a control compound because clearly it could not form a coordination complex with $1 \mathrm{~b}$. 
The ${ }^{1} \mathrm{H}$ NMR spectrum of $1 \mathrm{~b}$ in $\mathrm{CDCl} 3$ showed broad proton signals (similar to $1 \mathrm{a}$ ) due to the dimerization process experienced by $\mathrm{Zn}$-salophen at millimolar concentration in non-polar solvents (vide supra). When increasing amounts of $2 a$ were added to the solution of $1 b$, the proton signals of the latter became sharper. No further changes were observed in the chemical shift values of $1 b$ when more than 1 equivalent of $2 a$ was added. These results indicated the disruption of the dimeric aggregates of $1 \mathrm{~b}$ and the formation a 1:1 coordination complex between $1 b$ and $2 a$ for which we assessed a binding constant higher than $10^{4} \mathrm{M}^{-1}$ in complete agreement with the previous estimate (Figure $\mathrm{S} 14 \mathrm{SI}$ ). In the early stages of the titration the proton signals corresponding to the pyridyl group of the naphthalimide 2a appeared upfield shifted compared to those of free 2a. Most likely, the magnetic anisotropy caused by the nearby $\mathrm{Zn}$-salophen unit in the coordination complex $1 \mathrm{~b} \bullet 2 \mathrm{a}$ are responsible for the observed upfield shift. ${ }^{24}$ A 2D-NOESY spectrum showed an intense cross peak between the protons $\alpha$ to the nitrogen atom of the pyridine ring in $2 \mathrm{a}$ and those of the tert-butyl group in the salophen $1 \mathrm{~b}$ (Figure 7) providing support to the formation of the $1 \mathrm{~b} \cdot 2 \mathrm{a}$ supramolecular complex with axial coordination geometry.

Figure 7.

The interaction of $1 \mathrm{~b}$ with $2 \mathrm{a}$ was also studied in chloroform using UV-Vis absorption spectroscopy. The coordination of the pyridine of $2 \mathrm{a}$ to the $\mathrm{Zn}$-salophen $1 \mathrm{~b}$ resulted in a slight increase and bathochromic shift of the absorption band at $420 \mathrm{~nm}(\Delta \lambda \sim 6 \mathrm{~nm}$, Figure S16 SI), which is the expected behavior for the formation of an axial pyridine:Zn-salophen coordination complex. ${ }^{23}$ The fit of the titration data to a 1:1 binding model was good and the calculated stability constant for the $1 \mathrm{~b} \cdot 2 \mathrm{a}$ complex was $K_{a}=(4.66 \pm 0.41) \times 10^{5} \mathrm{M}^{-1}$.

An analogous titration experiment performed with the reference compound $2 \mathrm{~b}$ did not produce noticeable changes in the absorption spectra of the Zn-salophen 1b (Figure S17 SI).

\section{Emission properties of the supramolecular complex $1 \mathrm{~b} \bullet 2 \mathrm{a}$}

The emission spectra of the symmetrically substituted $Z n$-salophen $1 \mathrm{~b}\left(\lambda_{\text {exc }}=360 \mathrm{~nm}\right)$ in chloroform solution displayed a weak band centered at $550 \mathrm{~nm}$ (Figure 6). In contrast, the separate emission spectra of compounds $2 a$ and $2 b$ showed strong bands with maxima at 420 and $430 \mathrm{~nm}$, respectively. ${ }^{25}$ The strong fluorescence shown by naphthalimides $2 \mathrm{a}$ and $2 \mathrm{~b}$ indicated that fluorescence quenching observed in 1a was not caused by the intervention of the triazole ring connector.

The interaction of the pyridyl derivative $2 \mathrm{a}$ with the symmetric $\mathrm{Zn}$-salophen $1 \mathrm{~b}$ was probed also by emission spectroscopy. For practical reasons, we performed a reverse titration, where $\mathrm{Zn}$-salophen $1 \mathrm{~b}$ was added to a $10^{-7} \mathrm{M}$ chloroform solution of naphthalimide $2 \mathrm{a}$. We observed an intense quenching of the emission band of $2 a$ as the amount of $1 b$ in solution was increased. Conversely, the incremental addition of $\mathrm{Zn}$-salophen $1 \mathrm{~b}$ to a solution of the reference naphthalimide $2 \mathrm{~b}$ had a negligible quenching effect on its emission. (Figure S18 SI).

Taken together, these results indicated that the quenching of the emission of the 1,8naphthalimide unit by the $\mathrm{Zn}$-salophen required a spatio-temporal proximity of the two 
chromophores, naphthalimide and Zn-salophen. Using steady-state fluorescence experiments is not possible to unequivocally assign the nature of the quenching process that takes place in the $1 \mathrm{~b} \cdot 2 \mathrm{a}$ supramolecular complex. Interestingly, we observed that the emission spectrum of a solution containing $1 \mathrm{~b}$ and $2 \mathrm{a}$ showed a slight increase in the emission band of the $\mathrm{Zn}$ salophen $(\lambda=550 \mathrm{~nm}$ ) compared to an analogous one containing $1 \mathrm{~b}$ and $2 \mathrm{~b}$ (Figure 8$)$. In addition, the absorption band of the $\mathrm{Zn}$-salophen $1 \mathrm{~b}$ centered at $420 \mathrm{~nm}$ perfectly overlaps the emission band of the 1,8-naphthalimide 2a (Figure S19 SI). Taken in concert, these observations suggested that the nature of the quenching process can be related to an energy transfer process. However, based on our results we cannot exclude the existence of a photoinduced electron transfer process as responsible of the quenching. ${ }^{25}$

The strong overlap that existed between the absorption spectra of the two chromophores did not allow the selective excitation of one of them. This limitation prevents the possibility to unequivocally demonstrate the energy transfer process.

Figure 8 .

In close analogy to the results obtained in the supramolecular $1 \mathrm{~b} \bullet 2 \mathrm{a}$ complex, we assigned the unexpected emission properties featured by the naphthalimide unit of the receptor $1 \mathrm{a}$ in chloroform solution to the existence of a photoinduced quenching process (ET or PET) with the $\mathrm{Zn}$-salophen moiety.

\section{Sensing studies}

Although the emission properties of receptor 1 a were not the expected ones, we decided to test its behavior in the presence of an anion using emission spectroscopy. Our expectation was that the binding of a coordinating anion to receptor 1 a could be transduced in changes in the emission properties of the naphthalimide unit (e.g. fluorescence restore). As shown in Figure 9a, the addition of 10 equivalents of TBA(AcO) to a micromolar solution of 1 a caused a hypsochromic shift of the emission band corresponding to the salophen unit. Unfortunately, the photoinduced process responsible for the quenching of the naphthalimide fluorescence in 1a (i.e. electron or energy transfer) was not strongly influenced by acetate binding.

Figure 9.

Finally, we studied the behavior of the supramolecular complex $1 \mathrm{~b} \bullet 2 \mathrm{a}$ as a "turn-on"fluorescent sensor-ensemble for the detection of anions. In this case, the addition of the acetate anion ( 0 to $10 \mathrm{eq}$ ) to the solution containing the partially assembled $1 \mathrm{~b} \bullet 2 \mathrm{a}$ complex caused an increase in the emission of the 1,8-naphthalimide unit (Figure 9b). This observation is in complete agreement with the displacement of the pyridine-functionalized naphthalimide $2 \mathrm{a}$ in the $1 \mathrm{~b} \cdot 2 \mathrm{a}$ complex by the acetate anion yielding the $1 \mathrm{~b} \bullet \mathrm{AcO}$ - complex. The reported association constant for $\mathrm{Zn}$-salophen $1 \mathrm{~b} \bullet \mathrm{AcO}$ - complex is one order of magnitude higher than the one we calculated for the $1 b \bullet 2 a$ complex.19 Therefore, acetate effectively competes with 
the pyridine-functionalized 1,8-naphthalimide bound to the $\mathrm{Zn}$-salophen. The free naphthalimide $2 \mathrm{~b}$ that is released to the bulk solution recovers its emission properties.

\section{Conclusions}

In conclusion, we have synthesized a Zn-salophen receptor, 1a, covalently decorated with a 1,8-naphthalimide unit. We have shown that the attachment of the $\mathrm{Zn}$-salophen to a 1,8naphthalimide provokes a significant quenching in the emission of the latter. We have also designed and characterized a supramolecular system based on the symmetric Zn-salophen $1 \mathrm{~b}$ and the 1,8-naphthalimide pyridyl derivative 2a. In chloroform the two components form a 1:1 complex in which the pyridyl residue is axially coordinated to the $\mathrm{Zn}$-salophen. By means of UV-Vis titrations, we determined the association constant of the $1 \mathrm{~b} \cdot 2 \mathrm{a}$ complex to be $K_{a}=$ $(4.66 \pm 0.41) \times 10^{5} \mathrm{M}^{-1}$. Interestingly, the emission of the naphthalimide $2 \mathrm{a}$ is also quenched upon formation of the $1 \mathrm{~b} \cdot 2 \mathrm{a}$ complex ${ }^{19}$. The results of the investigation of the emission properties of the supramolecular system $1 \mathrm{~b} \bullet 2$ a suggested that a photoinduced energy transfer process could be responsible for the quenching of the naphthalimide unit. We ascribed the existence of a similar photoinduced process in the covalent structure of receptor 1a to explain the observed quenching of the fluorophore component. Unfortunately, the performed steadystate fluorescent experiments were not suitable to discard the existence of a photoinduced electron transfer between chromophores as an alternative pathway for emission deactivation.

The $\mathrm{Zn}$-salophen receptor 1a equipped with the naphthalimide unit transduces the binding of acetate anions to the $\mathrm{Zn}$-center in significant changes of the UV-Vis absorption spectrum. Conversely, the emission properties of $1 \mathrm{a}$ are almost unaffected by the recognition event. Finally, we used the supramolecular system $1 \mathrm{~b} \bullet 2 \mathrm{a}$ as a "turn-on"-fluorescent sensor ensemble in which the presence of the anion was signaled through a typical indicator displacement assay.

\section{Experimental Section}

\section{General Methods and Instrumentations}

Reagents and solvents were obtained from commercial suppliers and used without further purification. Spectrophotometric grade chloroform containing amylene as stabilizer was purchased from Sigma Aldrich and freshly deacidified with basic aluminum oxide before each UV-Vis measurement. The ${ }^{1} \mathrm{H}$ and ${ }^{13} \mathrm{C}$ NMR spectra were recorded at $300 \mathrm{MHz}, 400 \mathrm{MHz}$ or 500 $\mathrm{MHz}$ for $1 \mathrm{H}$ or at $75 \mathrm{MHz}, 100 \mathrm{MHz}$ and $125 \mathrm{MHz}$ for ${ }^{13} \mathrm{C}$, respectively. The chemical shifts ( $\delta$ ) for ${ }^{1} \mathrm{H}$ and ${ }^{13} \mathrm{C}$ are given in ppm relative to residual signals of the solvents $\left(\mathrm{CHCl}_{3}, \delta=7.26 \mathrm{ppm}\right.$ for ${ }^{1} \mathrm{H}$ NMR, $\delta=77.16 \mathrm{ppm}$ for ${ }^{13} \mathrm{C}$ NMR or DMSO, $\delta=2.50 \mathrm{ppm}$ for ${ }^{1} \mathrm{H}$ NMR, $\delta=39.52 \mathrm{ppm}$ for 13C NMR). When necessary, ${ }^{1} \mathrm{H}$ and ${ }^{13} \mathrm{C}$ signals were assigned by means of COSY, HSQC, NOESY and ROESY 2D-NMR sequences. High-resolution mass spectra (HRMS) were obtained on MicroTOF II from Bruker Daltonics (HPLC-MS-TOF) with positive ionization mode (ESI+). UV-Vis measurements were carried out on a Shimadzu UV-2401PC spectrophotometer equipped with a photomultiplier detector, double beam optics, and D2 and W light sources. Fluorescence 
measurements were performed in a Spectrofluorimeter Fluorolog Horiba Jobin Yvon. Dilute solutions $(A<0.05)$ were prepared in order to minimize inner filter effects.

Fluorescence quantum yield for naphthalimide 2a was determined by using quinine sulphate in $0.1 \mathrm{~N}(0.05 \mathrm{M})$ sulphuric acid solution as reference standard $(\Phi=0.53) .{ }^{28}$

The determination of the unknown quantum yields was performed by comparing the absorption and the integrated fluorescence intensity of solutions whose absorbance is maintained below 0.05 at the excitation wavelength $(350 \mathrm{~nm})$. The unknown quantum yield was then calculated applying Eq 1.

\section{Acknowledgements}

A.D.C, M.P. and F.Y.M. acknowledge "Ricerca scientifica di Ateneo 2013" and MIUR "PRIN 2010CX2TLM". M.P., G.A. and P. B. thank Gobierno de España MINECO (project CTQ201123014), Severo Ochoa Excellence Accreditation 2014-2018 (SEV-2013-0319), and the ICIQ Foundation for funding. We also acknowledge the support and sponsorship provided by COST Action CM1005.

Keywords: zinc-salophen $\bullet$ 1,8-naphthalimide $\bullet$ fluorescence quenching $\bullet$ energy transfer $\bullet$ indicator-displacement assay

${ }^{1}$ P. A. Gale, N. Busschaert, C. J. E. Haynes, L. E. Karagiannidis, I. L. Kirby, Chem. Soc. Rev. 2014, 43, $205-241$

M. Boiocchi, M. Licchelli, M. Milani, A. Poggi, D. Sacchi, Inorg. Chem. 2015, 54, 47-58.

${ }^{1}$ A. Schaly, R. Belda, E. Garcia-Espana, S. Kubik, Org. Lett. 2013, 15, 6238-6241.

${ }^{1}$ P. A. , Gale; C., Caltagirone, Anion sensing by small molecules and molecular ensembles. Chem. Soc. Rev. 2015, 00 (00), 0000-0000 DOI: $10.1039 / C 4 C S 00179 F$.

${ }^{1}$ X. D. Lou, D. X. Ou, Q. Q. Li, Z. Li, Chem. Commun. 2012, 48, 8462-8477.

${ }^{1}$ A. P. de Silva, B. McCaughan, B. O. F. McKinney, M. Querol, Dalton Trans. 2003, 1902-1913.

1 M. H. Lee, J. S. Kim and Jonathan L. Sessler, Small molecule-based ratiometric fluorescence probes for cations, anions, and biomolecules. Chem. Soc. Rev. 2015, 00 (00), 0000-0000 DOI: 10.1039/C4CS00280F (Highlight)

${ }^{1}$ Y. Zhou, Z. Xu, J. Yoon, Chem. Soc. Rev. 2011, 40, 2222-2235.

${ }^{1}$ M. E. Moragues, R. Martinez-Manez, F. Sancenon, Chem. Soc. Rev. 2011, 40, 2593-2643.

${ }^{1}$ A. Dalla Cort, P. De Bernardin, G. Forte, F. Y. Mihan, Chem. Soc. Rev. 2010, 39, 3863-3874

${ }^{1}$ K. L. Kuo, C. C. Huang, Y. C. Lin, Dalton Trans. 2008, 3889-3898.

${ }^{1}$ K. H. Chang, C. C. Huang, Y. H. Liu, Y. H. Hu, P. T. Chou, Y. C. Lin, Dalton Trans. 2004, 1731-1738.

${ }^{1}$ R. M. Duke, E. B. Veale, F. M. Pfeffer, P. E. Kruger, T. Gunnlaugsson, Chem. Soc. Rev. 2010, 39, 3936-3953.

${ }^{1}$ Z. C. Xu, K. H. Baek, H. N. Kim, J. N. Cui, X. H. Qian, D. R. Spring, I. Shin, J. Yoon, J. Am. Chem. Soc. 2010, 132, 601-610.

A. J. Moro, P. J. Cywinski, S. Korsten, G. J. Mohr, Chem. Commun. 2010, 46, 1085-1087.

${ }^{1}$ J. F. Zhang, S. Kim, J. H. Han, S. J. Lee, T. Pradhan, Q. Y. Cao, S. J. Lee, C. Kang, J. S. Kim, Org. Lett. 2011, 13, 5294-5297.

${ }^{1}$ A. W. Kleij, M. Kuil, M. Lutz, D. M. Tooke, A. L. Spek, P. C. J. Kamer, P. W. N. M. van Leeuwen, J. N. H. Reek, Inorg. Chim. Acta 2006, 359, 1807-1814.

${ }^{1}$ The hydrodynamic radius $\left(r_{\text {exp }}\right)$ was calculated from the experimental diffusion coefficient $(D)$ using the Stokes Einstein relation $(1)$.

$r_{\text {sph }}=k_{B} T / 6 \pi n D$

Where $k_{B}$ is the Boltzmann constant, $T$ is the absolute temperature and $\eta$ is the viscosity of the medium.

${ }^{1}$ S. J. Wezenberg, E. C. Escudero-Adan, J. Benet-Buchholz, A. W. Kleij, Chem. Eur. J. 2009, 15, 5695-5700.

${ }^{1}$ A. D. Cort, P. De Bernardin, L. Schiaffino, Chirality 2009, 21, 104-109.

${ }^{1}$ G. Consiglio, S. Failla, P. Finocchiaro, I. P. Oliveri, R. Purrello, S. Di Bella, Inorg. Chem. 2010, 49, 5134-5142.

${ }^{1}$ M. E. Germain, T. R. Vargo, P. G. Khalifah, M. J. Knapp, Inorg. Chem. 2007, 46, 4422-4429.

${ }^{1}$ A. Dalla Cort, L. Mandolini, C. Pasquini, K. Rissanen, L. Russo, L. Schiaffino, New J. Chem. 2007, 31, 1633-1638.

${ }^{1}$ E. C. Escudero-Adan, J. Benet-Buchholz, A. W. Kleij, Eur. J. Inorg. Chem. 2009, 3562-3568.

${ }^{1}$ In order to compare the emission intensity of all the molecules under study (1a, $\mathbf{1} \mathbf{b}$ and $\mathbf{2 a}$ or $\mathbf{2} \mathbf{b}$ ), we selected an excitation wavelength at which all the molecules are excited.

${ }^{1}$ The relative energy levels of the frontier HOMO and LUMO orbitals of $\mathbf{1 b}$ and $\mathbf{2 a}$ were calculated at the B3LYP/6-31G theory level using a chloroform continuum solvent model PCM. The computed energy values were HOMO(1b): $-5.32 \mathrm{eV}, \mathrm{LUMO}(\mathbf{1 b}):-2.03 \mathrm{eV}$, $\mathrm{HOMO}(2 \mathrm{a}):-6.39 \mathrm{eV}$ and $\operatorname{LUMO}(\mathbf{2 a}):-2.70 \mathrm{eV}$. These energy values suggested that a photoinduced electron transfer process occurring from the HOMO of $\mathbf{1 b}$ to the SOMO-1 of $\mathbf{2 a}$ is thermodynamically viable. However, the results of these calculations were 
not sufficient to rule out the existence of an energy transfer process as an alternative mechanism of the emission quenching of $\mathbf{2 a}$ in the $\mathbf{1 b \cdot 2 a}$ complex.

${ }^{1}$ The excitation wavelength was fixed at $310 \mathrm{~nm}$ in order to have a minimum direct excitation of the 1,8-naphthalimide units (2a or $\mathbf{2 b}$ ). This allowed to observe the emission band of the Zn-salophen $\mathbf{1 b}$, otherwise covered by the strong emission of the 1,8naphthalimides.

1 M. J. Adams, J. G. Highfield, G. F. Kirkbright, Anal. Chem. 1977, 49, 1850-1852. 
Figure 1. Line-drawing structures of the molecules prepared and studied in this work.

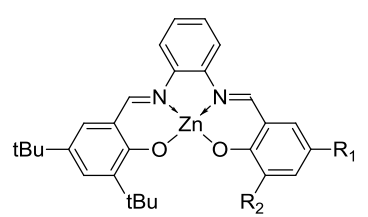

$1 \mathrm{a} ; \mathrm{R}_{1}=$

$1 b ; R_{1}=R_{2}=t B u$
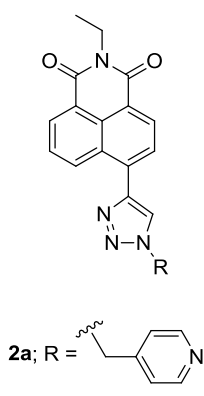

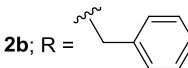

Scheme 1. Synthesis of non-symmetrical salophen 1a. Reagents and conditions: a) $\left[\mathrm{Cu}\left(\mathrm{CH}_{3} \mathrm{CN}\right)_{4}\right] \mathrm{PF} 6$, TBTA, DMSO, room temperature, $48 \mathrm{~h}, 69 \%$; b) $\mathrm{ZnCl}_{2}, \mathrm{Et}_{3} \mathrm{~N}, \mathrm{DCM} / \mathrm{MeOH} 2: 1$, room temperature, 18 h, 75\%.
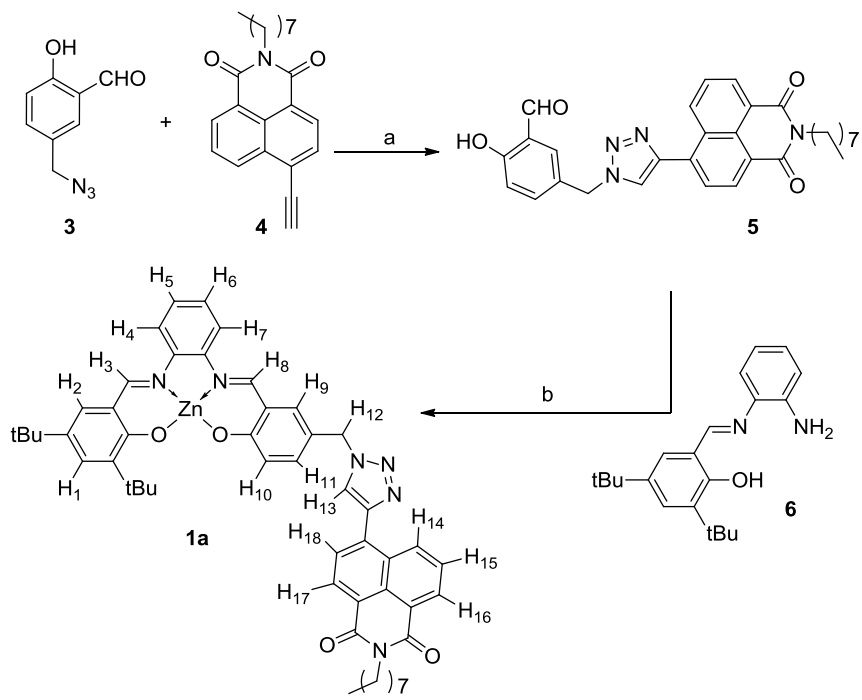
Figure 2. Top (a) and side (b) views of the MM3 energy minimized structure of salophen 1a shown in stick representation. Non-polar hydrogen atoms are omitted for clarity. A sphere centered on 1a and with a radii of $8 \AA$ that corresponds to the value determined from the DOSY experiment is shown in (b).

a)

b)
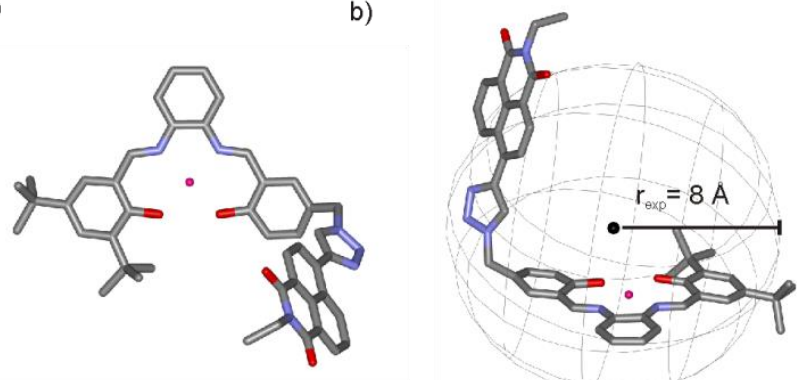

Figure 3. Selected region of the ${ }^{1} \mathrm{H}-\mathrm{NMR}$ of $\mathbf{1 a}$ in $\mathrm{CDCl}_{3}\left(4.49 \times 10^{-3} \mathrm{M}\right)$ upon addition of 0 (a), 0.5 (b), 1 (c) and 1.5 (d) equivalent of $\operatorname{TBA}(\mathrm{AcO})$.

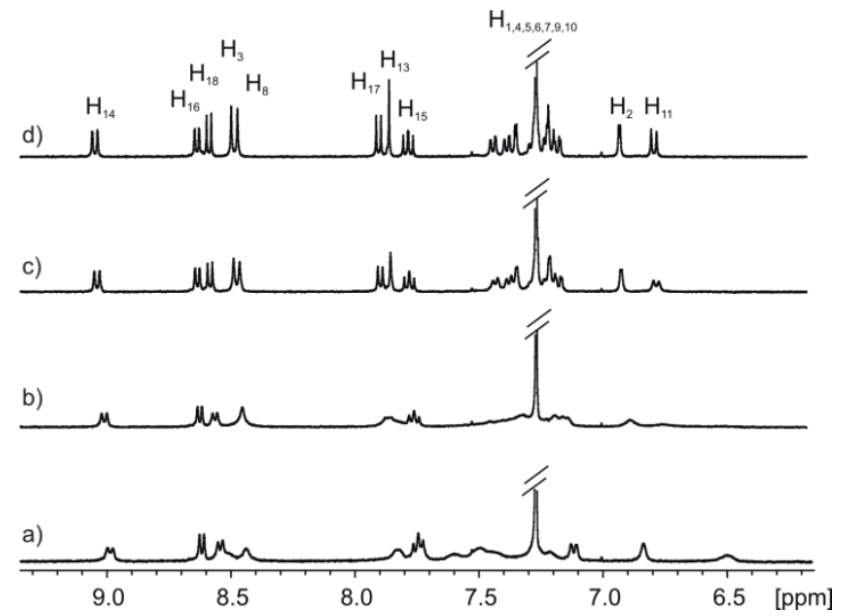

Figure 4. Absorption spectra in molar absorption coefficient scale of $\mathbf{1 a}$ (solid line), $\mathbf{1 b}$ (dotted line) and $\mathbf{2 b}$ (dashed line) in chloroform.

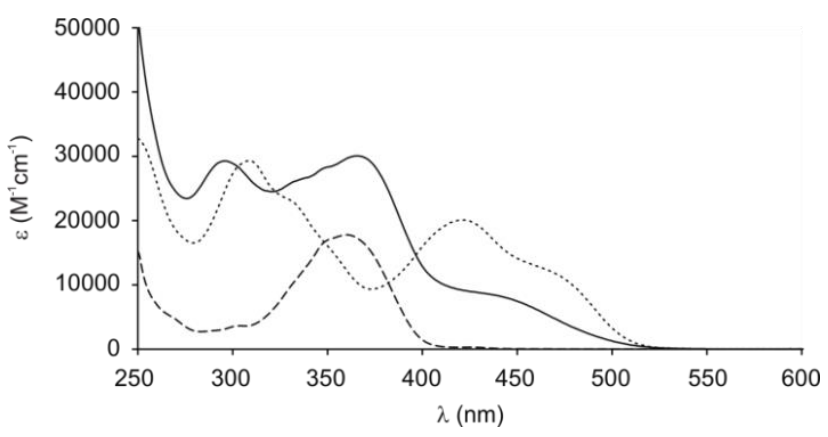


Figure 5. Absorption spectra of $1 \mathbf{a}\left(3.69 \times 10^{-5} \mathrm{M}\right)$ upon addition of incremental amounts of $\mathrm{AcO}^{-}(0-6$ eq) in chloroform. Inset: fit of the experimental data at $405 \mathrm{~nm}$ and $370 \mathrm{~nm}$ to the calculated theoretical binding curve for a 1:1 binding model considering two coloured species (free 1a and 1a*AcO' complex).

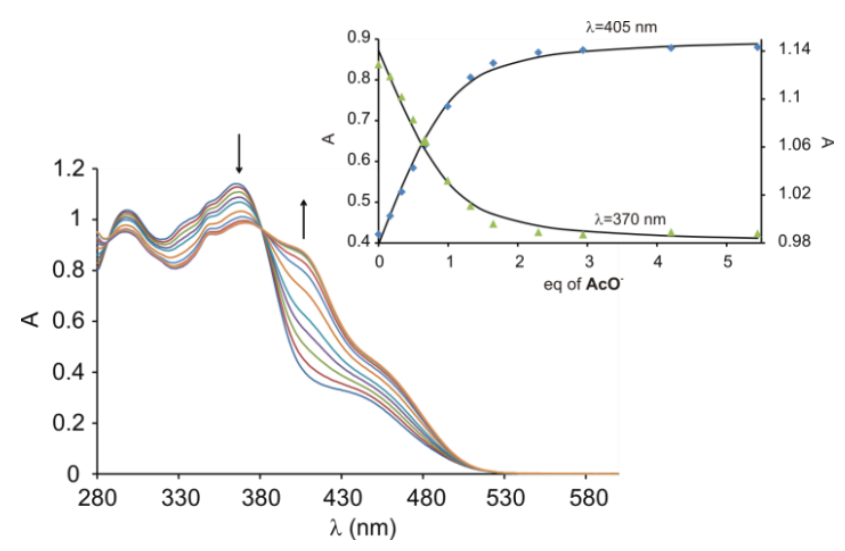

Figure 6. Emission spectra of $\mathbf{1 a}$ (solid line, $1 \times 10^{-6} \mathrm{M}$ ), $\mathbf{1 b}$ (dotted line, $1 \times 10^{-6} \mathrm{M}$ ) and $\mathbf{2 b}$ (dashed line, $1 \times 10^{-7} \mathrm{M}$ ) in chloroform $\lambda_{\mathrm{ex}}=360$ $\mathrm{nm}$. Please notice the break in the scale of normalized emission intensity.

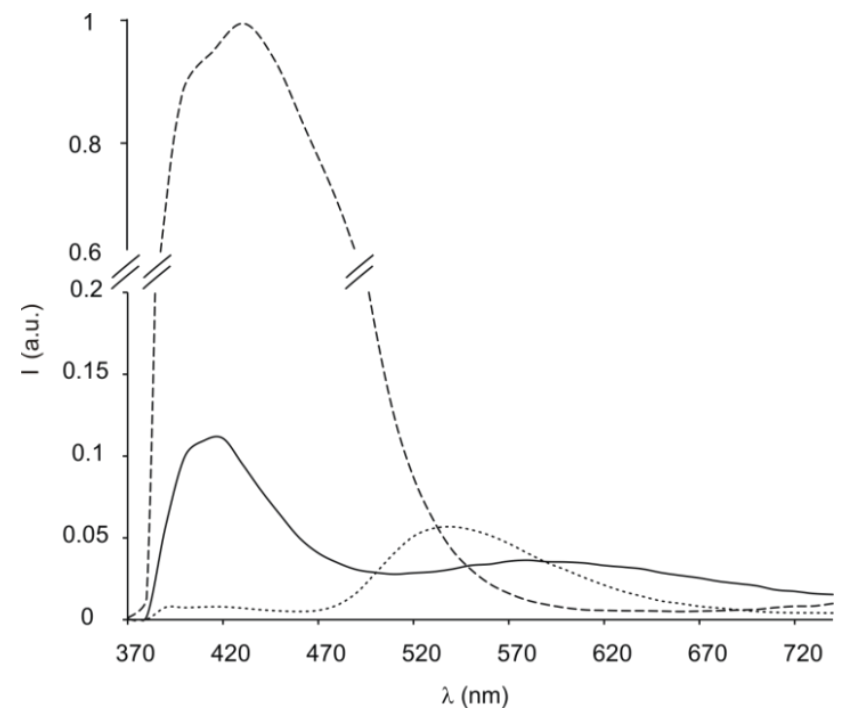

Figure 7. Selected area of the 2D NOESY spectrum of the $1: 1$ complex $\mathbf{1} \mathbf{b} \cdot \mathbf{2 a}$ in $\mathrm{CDCl}_{3}$ solution. The MM3 energy minimized structure of the $\mathbf{1 b} \cdot \mathbf{2 a}$ complex is shown as an inset.

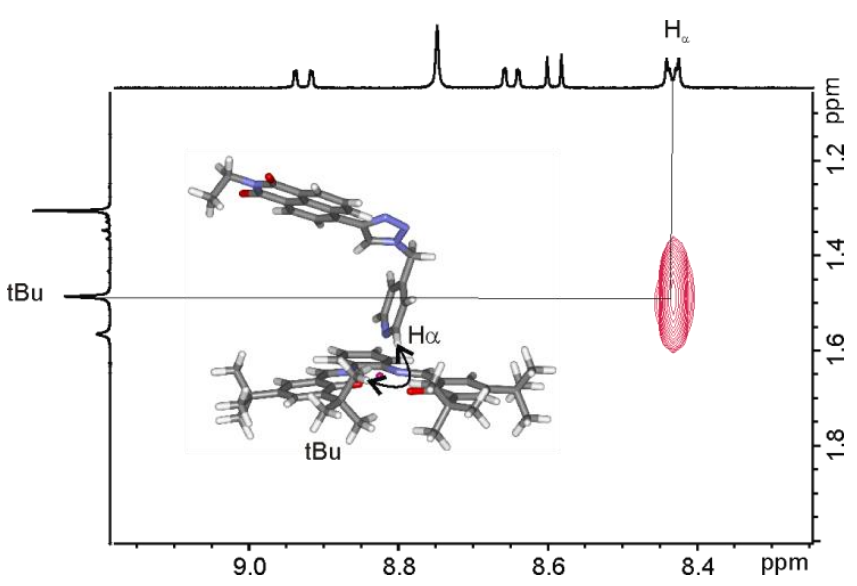


Figure 8. Comparison of the emission spectra at the final point of the titration of a solution of $2 \mathbf{a}$ (red line, $6.62 \times 10^{-7} \mathrm{M}$ ) and $2 \mathbf{b}$ (green line, $6.84 \times 10^{-7} \mathrm{M}$ ) with $11 \mathrm{eq}$ of $\mathbf{1 b} . \lambda_{\mathrm{ex}}=310 \mathrm{~nm}$.

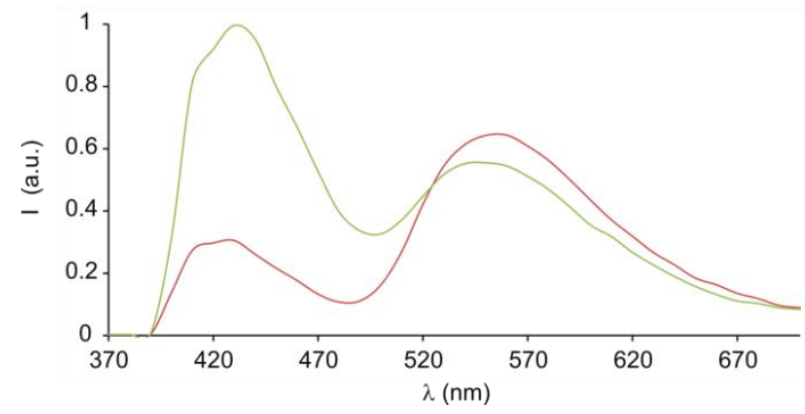

Figure 9. (a) Changes observed in the emission spectra of $1 \mathrm{a}\left(1.51 \times 10^{-6} \mathrm{M}\right)$ in chloroform solution upon addition of acetate $\left(0-10\right.$ eq). $\lambda_{\mathrm{ex}}$ $=380 \mathrm{~nm}$. (b) Recovery of fluorescence emission upon addition of acetate anion (as tetrabutylammonium salt, 0-10 eq) to a chloroform solution of $\mathbf{2 a}\left(8.29 \times 10^{-6} \mathrm{M}\right)$ and $\mathbf{1 b}\left(1.75 \times 10^{-5} \mathrm{M}\right) . \lambda_{\mathrm{ex}}=360 \mathrm{~nm}$.

a)

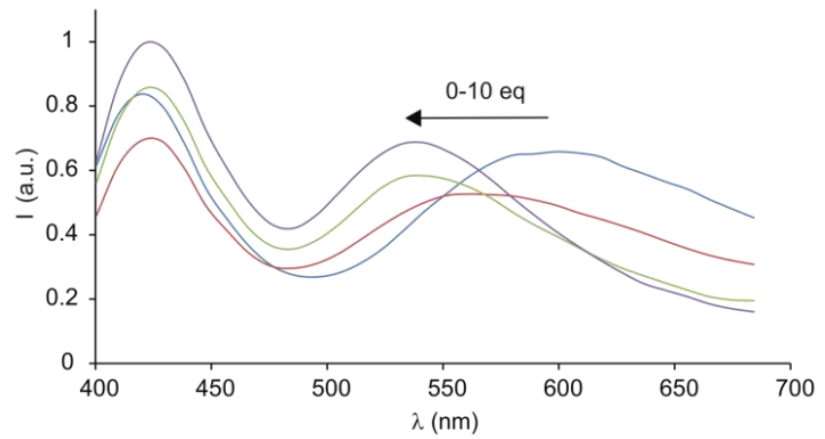

b)

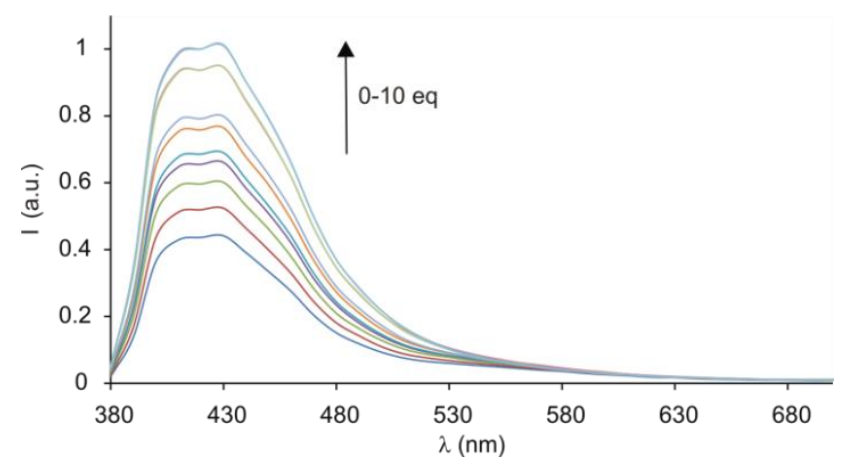




$$
\Phi_{X}=\Phi_{S T}\left(\frac{\operatorname{grad}_{X}}{\operatorname{grad}_{S T}}\right)\left(\frac{n^{2} X}{n^{2}}\right)
$$

Where $X$ and $S T$ are the sample and the standard, respectively; $\Phi$ is the fluorescence quantum yield; grad is the gradient of the plot of the integrated fluorescence intensity vs absorbance; and $n$ is the refractive index of the solvent $\left(X=\mathrm{CHCl}_{3} n=1.45, S T=\right.$ $\mathrm{H}_{2} \mathrm{SO}_{4} 0.1 \mathrm{~N}, n=1.35$ ). 